\title{
Economic impact of dry eye disease in Spain: a multicentre retrospective insurance claims database analysis
}

Josep Darbà Phd $^{1 *}$, Meritxell Ascanio MSc ${ }^{2}$

${ }^{1}$ Universitat de Barcelona, Barcelona, Spain; ${ }^{2}$ BCN Health Economics \& Outcomes

Research S.L., Barcelona, Spain

Running title: Economic impact of dry eye disease in Spain

*Corresponding Author:

Josep Darbà

Department of Economics

Universitat de Barcelona

Diagonal 696

Barcelona 08034, Spain

Tel. +34 934020110 / + 34934021937

Fax +34 934039082

E-maildarba@ub.edu 


\section{ABSTRACT}

Purpose: To analyse the occurrence and cost of dry eye disease in Spain throughout recent years.

Methods: A cross-sectional analysis based on anonymised data recorded from 1997 to 2015 from public and private hospitals and healthcare centres from an insurance claims database with a total of 36,081 patients eligible for the study after duplicate elimination. Selection from the database by means of the International statistical classification of diseases and related health problems (9th revision) associated with Dry eye syndrome codes. Number of cases, total cost, mean cost per patient, annual number of patients, annual number of visits, patient characteristics, hospitalizations and comorbidities.

Results: Only $12 \%$ of the patients who presented dry eye diseased were male, whereas the mean age was approximately 65 across all study groups. Both the annual number of patients and visits have increased exponentially since 1997 raising from 1,079 and 1,342 to 3,097 and 5,922 patients and visits, respectively. Hospitalizations had a length of stay of 9.6 [SD \pm 11.6$]$ days where more than $65 \%$ were due to emergency admissions and $92 \%$ were discharged home. Total costs were found to increase from 3.7M€ to $30.3 \mathrm{M} €$ during the studied time period and an increase was also observed concerning the mean cost per patient and per visit.

Conclusions: Estimating that disease incidence is likely to increase due to the influence of modern day work place, it is important to take into account the high economic burden and large decrease in quality of life in regards to Spanish society and health policies.

\section{KEYWORDS}

Dry eye disease; economic impact study; claims database analysis; Spain. 


\section{INTRODUCTION}

The currently established definition of dry eye disease (DED) describes it as a chronic and progressive multifactorial disorder characterized by a tear film and ocular surface alteration [1-3]. The cause of this phenomenon may be either tear deficiency or excessive evaporation of the lacrimal fluid, which may cause ocular discomfort, irritation, ocular fatigue, visual disturbance and tear film instability, ultimately leading to potential damage to the ocular surface. An increased osmolarity of the tear film and inflammation of the ocular surface can also be observed. All these factors contribute to a noticeable decrease in quality of life (QoL) [4].

Risk factors associated to DED include adverse environmental conditions such as lack of humidity, chemicals and atmospheric dust, prolonged computer and electronic device usage, contact lenses and ionizing radiation. It is therefore very closely associated to modern day offices and will only lead to an incidence increase throughout the years [2-5]. According to the cause of tear film defect, DED can be subcategorized into Sjögren syndrome (SS) DED and non-SS DED in the case of tear deficiency [6]. In regards to excess evaporation, the most common cause is meibomian gland dysfunction (MGD) [7]. The importance of knowing the number of cases, cost and epidemiology of the disease lies in the fact that DED is among the most frequently diagnosed ophthalmologic diseases and leads to a large economic and social burden by means of a decrease in patient QoL [2]. The impact is associated to both the patients and the healthcare system due to direct and indirect costs concerning medical visits, therapies, surgeries, the frequent use of eye drops and a decrease in professional productivity caused by a reduced QoL [7]. 
Many studies indicate that the occurrence of the disease increases with age and is more commonly diagnosed in women $[1-5,8]$. However, reports disagree when it comes to the prevalence range. While some imply DED affects from $5 \%$ to $33 \%$ of the worldwide population [7], others argue it can reach as much as $50 \%$ or as little as $0.02 \%[1,9]$. A second fact that most publications acknowledge is that DED has a higher prevalence in Asiatic or Far Eastern countries relative to Europe or the US. The most common hypothesis being, the existence of a genomic factor associated to the disease $[5,10]$. Because of its close association to the work environment, disease occurrence is expected to increase during the following years generating more costs for both health service and patients.

There is a clear lack of research regarding the number of cases and cost of DED, however it is apparent that the economic burden of the disease is not negligible.

The most recent and seemingly the only publication regarding dry eye disease occurrence in Spain is from 2009 [8]. The article discusses the epidemiology of DED in over 40 year old patients in the north-western region of the country exclusively. A second publication by the same authors (2012) evaluates the occurrence of MGD, a subcategory of DED, in the same region [11].

A comparative study discusses the occurrence and annual costs of DED in France, Germany, Italy, Spain, Sweden and the United Kingdom amongst patients managed by ophthalmologists [9]. However, there are no other references available regarding DEDrelated costs. Consequently, there is an obvious need to update the available information regarding DED. 
Herein we study the number of cases and cost of DED in Spain throughout recent years by means of the analysis of data obtained from an insurance claims databases containing private and public hospital and healthcare centre records concerning the whole territory.

\section{METHODS}

A total of 62,050 records of patients with DED were obtained from the database by means of the appropriate International statistical classification of diseases and related health problems, 9th revision (ICD9) code. The national hospitalisation database contains information regarding records generated from both private and public hospitals and health centres available since 1997 up to 2015, on account of recent reports not yet being published. Parameters such as health centres and medical history identifiers have been recoded in order to maintain records anonymised.

After discarding duplicate data, corresponding to patients that have been visited more than once, the final analysed data consisted of 36,081 records, which are the number of patients that have developed the pathology at some point in time.

Ethics Committee ruled that approval was not required for this study.

Data analysis

Data presentation and analysis are generally descriptive, having not tested any specific hypothesis. Data is presented as mean values \pm standard deviation (SD) and as percentages.

The number of visits and number of patients per year has been calculated. Patient characteristics and most common comorbidities have also been studied.

\section{RESULTS}


In order to obtain patients records for DED five ICD9 codes were used. The codes that were considered corresponded to vitamin A deficiency with xerophthalmic scars of cornea (ICD9 264.6), Xerophthalmia due to vitamin A deficiency (ICD9 264.7), Keratoconjunctivitis sicca not specified as Sjögren's (ICD9 370.33), Dry eye syndrome (ICD9 375.15) and Keratoconjunctivitis sicca Sjögren's disease (ICD9 710.2). All the above diseases fall under the scope of DED.

Out of a total of 36,081 patients around 4,308 (12\%) were males and 31,769 (88\%) female (Table 1). Both sex groups had similar means in regards to age, approximately 65 years with a SD of 15.24 years concerning the male group and 15.06 years for the women.

Table 1 Dry eye disease database patient characteristics

\begin{tabular}{cllll} 
& All patients & Men & Women & Undetermined \\
\hline Number of patients & 36,081 & 4,308 & 31,769 & 4 \\
Age (years) & & $(11.94 \%)$ & $(88.05 \%)$ & $(0.01 \%)$ \\
Mean & 65.56 & 65.15 & 65.61 & 63.25 \\
SD & 15.24 & 16.48 & 15.06 & 11.32 \\
Median & 68 & 68 & 68 & 59 \\
Range & $0-105$ & $0-98$ & $0-105$ & $55-80$
\end{tabular}

Since records began, cases of patients with DED have shown an exponential growth throughout the years therefore, as expected, health service visits also show an annual increase (Table 2). This is partially explained by the chronic nature of the disease. 
However, visits per year increase more abruptly as a result of an increment of patients that have been visited more than once or an increase of visits per patient.

Hospitalizations have a mean duration of stay of 9.6 days, and 8,430 out of the recorded 62,050 visits (13.6\%) are readmissions (Table 3). Most hospital admissions, regarding both inpatient (day care) and outpatient hospitalisations (at least 1 night stay), are due to emergencies $(65.5 \%)$ whereas $34.2 \%$ are scheduled. Regarding the nature of hospital discharges, patients are most frequently sent home (92.4\%), a minimal portion of them are transferred to another hospital $(1.9 \%)$ or are discharged voluntarily $(0.2 \%)$.

Table 2 Patients per year, visits per year and cost analysis related to dry eye disease

\begin{tabular}{cccccc} 
Year & Patients & Visits & $\begin{array}{c}\text { Total Cost } \\
\text { (€) }\end{array}$ & $\begin{array}{c}\text { Mean cost } \\
\text { per visit (€) }\end{array}$ & $\begin{array}{c}\text { per patient } \\
(\boldsymbol{\Theta})\end{array}$ \\
\hline $\mathbf{1 9 9 7}$ & 1,079 & 1,342 & $3,667,846$ & 2,733 & 3,399 \\
$\mathbf{1 9 9 8}$ & 1,181 & 1,634 & $4,815,757$ & 2,947 & 4,078 \\
$\mathbf{1 9 9 9}$ & 1,135 & 1,720 & $4,875,330$ & 2,834 & 4,295 \\
$\mathbf{2 0 0 0}$ & 1,285 & 1,905 & $5,420,442$ & 2,845 & 4,218 \\
$\mathbf{2 0 0 1}$ & 1,257 & 2,139 & $6,492,870$ & 3,035 & 5,165 \\
$\mathbf{2 0 0 2}$ & 1,358 & 2,263 & $7,935,453$ & 3,507 & 5,843 \\
$\mathbf{2 0 0 3}$ & 1,567 & 2,643 & $9,820,864$ & 3,716 & 6,267 \\
$\mathbf{2 0 0 4}$ & 1,631 & 2,627 & $10,368,505$ & 3,947 & 6,357 \\
$\mathbf{2 0 0 5}$ & 1,693 & 2,795 & $11,498,933$ & 4,114 & 6,792 \\
$\mathbf{2 0 0 6}$ & 1,669 & 2,884 & $11,594,625$ & 4,020 & 6,947 \\
$\mathbf{2 0 0 7}$ & 1,744 & 3,111 & $13,031,498$ & 4,189 & 7,472 \\
$\mathbf{2 0 0 8}$ & 2,091 & 3,369 & $15,832,045$ & 4,699 & 7,572 \\
$\mathbf{2 0 0 9}$ & 2,189 & 3,771 & $18,016,603$ & 4,778 & 8,231 \\
$\mathbf{2 0 1 0}$ & 2,327 & 4,128 & $21,267,904$ & 5,152 & 9,140 \\
$\mathbf{2 0 1 1}$ & 2,417 & 4,389 & $23,347,643$ & 5,320 & 9,660 \\
$\mathbf{2 0 1 2}$ & 2,599 & 4,612 & $23,840,733$ & 5,169 & 9,173 \\
& & & & &
\end{tabular}




\begin{tabular}{cccccc}
$\mathbf{2 0 1 3}$ & 2,859 & 5,191 & $26,099,659$ & 5,028 & 9,129 \\
$\mathbf{2 0 1 4}$ & 2,903 & 5,605 & $26,731,792$ & 4,769 & 9,208 \\
$\mathbf{2 0 1 5}$ & 3,097 & 5,922 & $30,286,815$ & 5,114 & 9,779 \\
\hline Total & - & - & $274,945,316$ & 77,918 & 132,726
\end{tabular}

Table 3 Dry eye disease-related hospitalizations

\begin{tabular}{llll} 
Hospitalizations & & & \\
\hline & $\begin{array}{l}\text { Duration of stay in } \\
\text { days (mean } \pm \text { SD) } \\
\text { Rdmission }\end{array}$ & $9.6 \pm 11.6$ & \\
& Readmissions & 8,430 & $13.6 \%$ \\
& Emergency & 40,666 & $65.5 \%$ \\
& Scheduled & 21,205 & $34.2 \%$ \\
Discharge & Other-unknown & 179 & $0.3 \%$ \\
\hline & Home & 57,338 & $92.4 \%$ \\
& Transfer to another & 1,176 & $1.9 \%$ \\
& Hospital & 129 & $0.2 \%$
\end{tabular}

The most common DED-associated comorbidities (Table 4) were related to hypertension in $31.7 \%$ of the cases, rheumatoid arthritis (15.7\%), unspecified lipidemias and hyperlipidemias (10.4\%), diabetes mellitus (10.1\%) and atrial fibrillation $(9.8 \%)$.

Table 4 Dry eye disease-associated comorbidities

\begin{tabular}{lll} 
ICD 9 code & Disease description & Visits (\%) \\
\hline $\mathbf{4 0 1 . 9}$ & Essential hypertension, unspecified & $19,666(31.7 \%)$ \\
\hline $\mathbf{7 1 4 . 0}$ & Rheumatoid arthritis & $9,771(15.7 \%)$ \\
\hline $\mathbf{2 7 2 . 4}$ & $\begin{array}{l}\text { Other unspecified hyperlipidemias and } \\
\text { lipidemias }\end{array}$ & $6,474(10.4 \%)$
\end{tabular}




\begin{tabular}{lll}
$\mathbf{2 5 0 . 0}$ & $\begin{array}{l}\text { Diabetes mellitus without mention of } \\
\text { complication }\end{array}$ & $6,253(10.1 \%)$ \\
\hline $\mathbf{4 2 7 . 3 1}$ & Atrial fibrillation & $6,074(9.8 \%)$
\end{tabular}

Total annual costs, mean costs per visit and mean cost per patient were calculated using the information provided within the hospital and health care centre database (Table 2). Mean costs per visit increase annually in a steady manner with slight oscillations and show a modest decrease from 2011 up to 2015 when the cost increments once again. Values range from $2,733.12 €(1997)$ to $5,114.29 €$ (2011) per visit. In regards to mean cost per patient, values are almost tripled within the 18 years of recorded data. The abrupt increase from 3,399.30€ in 1997 to $9,779.40 €$ in 2015 is also due to the increase of visits per patient.

Taking this into account and the fact that a larger number of patients and visits are recorded, it is only expected that total cost of DED also grows annually, increasing more than 8 times over a period of 19 years. This escalates the total annual cost from $3.7 \mathrm{M} €$ in 1997 to $30.3 \mathrm{M} €$ in 2015 .

\section{DISCUSSION}

It is generally difficult to measure the number of cases of DED in the general population due to a series of aspects: first of all, the multifactorial nature of the disease but also scarce research and differential definitions of the disease, which the 2007 Report of the International Dry Eye Workshop (DEWS) attempted to address [4]. Additionally, according to the severity of the disease patients might opt to self-treat instead of visiting a health care centre and therefore are not accounted for in the databases. 
The results described in this article are within the scope of most past publications in regards to DED occurrence. Spanish DED number of cases calculated from 2006 to 2015 are contained within the normal range and are in accordance with previous measurement regarding the north-western region of the country [8]. Results also agree with the fact that the disease is age-associated and more common in women.

However literature regarding the cost of DED in France, Germany, Italy, Spain, Sweden and the United Kingdom suggest the disease has an occurrence of $0.03 \%$ in Spain [9]. Nevertheless, the value was calculated by means of the information provided by a survey addressed at consultant ophthalmologists who might have only treated the most severe cases of the disease. This particular study therefore excludes self-treated patients and patients treated by general practitioners and generalist ophthalmologists, which may explain the inconsistent results.

The Salnes Eye Study in particular studied a total of 654 subjects from the north-western region of Spain, 37.2\% of them males, with a mean age of 63.6 [SD 14.4] years, ranging from 40 to 96 [8]. In comparison, the present study accounts for 36081 patients from across the whole country, $11.94 \%$ of them males, with a similar age mean of 65.56 [SD 15.24] ranging from 0 to 105 years.

Whereas the 2009 Salnes Eye Study only found rosacea to be associated to DED, the 2012 publication concerning symptomatic and asymptomatic MGD prevalence also describes diabetes and cardiovascular disease as the most common comorbidities for asymptomatic MGD and rosacea and rheumatoid arthritis in regards to symptomatic MGD. Our research agrees considering diabetes mellitus and rheumatoid arthritis as two 
of the most frequent comorbidities and adds hypertension, hyperlipidemias and lipidemias and atrial fibrillation to the list [11].

Previously published data regarding DED-related annual costs is based on the information obtained from surveying ophthalmologists between the years 2003 and 2004 . The 2009 report estimated the annual burden per patient for six European countries: UK (approx. 1,100 USD), Spain (approx. 800 USD), Italy (approx. 600 USD), Germany (approx. 500 USD), Sweden (approx. 400 USD), and France (approx. 300 USD) [9].

A direct comparison is not feasible, however, considering the herein calculated mean costs per patient and per visit during the years 2003 and 2004, when data was collected for the Clegg et al. study, large discrepancies are observed. Values of 6,267.30 € in 2003 and $6,357.15 €$ in 2004 per patient are obtained whereas a much lower cost is considered in the previous publication.

This might be the case due to the use of very distinctive data acquisition methodologies: in one hand, acquired from healthcare records from hospitals and healthcare centres and on the other, based on ophthalmologist surveys.

\section{CONCLUSIONS}

The analysis of total hospitalisation records of DED in Spain indicates that DED-related hospitalisations entailed a mean cost per patient of 9,779.40€ in 2015.

Estimated DED incidence is likely to increase due to the influence of modern day work place and lifestyle. For that reason, it is important to take into account the high economic burden and large decrease in QoL in regards to Spanish society and health policies.

\section{LIST OF ABBREVIATIONS}

DED: dry eye disease 
QoL: quality of life

SS: Sjögren syndrome

MGD: meibomian gland dysfunction

ICD9: International statistical classification of diseases and related health problems, 9th revision

SD: standard deviation

DEWS: Dry Eye Workshop

\section{DECLARATIONS}

\section{Acknowledgements}

Not applicable

\section{Declaration of ethics}

Ethics Committee declared that ethics approval was not required for this study.

\section{Declaration of interest}

The authors have no conflicts of interest to declare.

\section{Funding}

The author(s) received no financial support for the research, authorship, and/or publication of this article.

\section{Author Contributions}

JD contributes to the investigation by interpreting the economic current situation of DED in Spain taking into account the introduction of the new treatment and was a major contribution in the intellectual content revision and gave final approval of the version to be published. MA analysed the current occurrence rate of DED in Spain, analysed and 
interpreted the statistical data and was a major contributor in writing the manuscript. All authors read and approved the final manuscript.

\section{REFERENCES}

1. Stapleton F, Alves M, Bunya VY. TFOS DEWS II Epidemiology Report. Ocul Surf. 2017; 15:334-365.

2. de Pinho Tavares F, Stehling Fernandes, Freitas Bernardes T, Alvim Bonfioli A, Carneiro Soares. Dry eye disease. Semin Ophthalmol. 2010;25(3):84-93.

3. Sharma RA, Mather R. Dry eye disease. Canadian Medical Association Journal. 2014; 14:186.

4. Lemp MA. 2007 Report of the International Dry Eye Workshop (DEWS). Ocul Surf. 2007; 5(2).

5. Vicenete-Herrero MT, Ramírez-Iñiguez de la Torre MV, Terradillos-García MJ, López González ÁA. Síndrome del ojo seco. Factores de riesgo laboral, valoración y prevención. SEMERGEN. 2014; 40(2):97-103.

6. Ramos-Casals M, Casanovas A, Oristrell J. Síndrome de Sjögren. Guía de tratamiento 2009. Guía de pràctica Clínica SEMI. 2009.

7. McDonald M, Patel DA, Keith MS, Snedecor SJ. Economic and humanistic burden of dry eye disease in Europe, North America, and Asia: A Systematic Literature Review. Ocul Surf. 2016;14(2):144-167.

8. Viso E, Rodriguez-Ares MT, Gude F. Prevalence of and Associated Factors for Dry Eye in a Spanish Adult Population (The Salnes Eye Study). Ophthalmic Epidemiol. $2009 ; 16: 15-21$.

9. Clegg JP, Guest JF, Lehman A, Smith AF. The Annual Cost of Dry Eye Syndrome in France, Germany, Italy, Spain, Sweden and the United Kingdom Among Patients Managed by Ophthalmologists. Ophthalmic Epidemiol. 2006; 13:263-274. 
10. Merayo Lloves J. Conceptos Actuales en Ojo Seco. Del Síndrome a la Enfermedad. Grupo Español de Superficie Ocular y Cornea (GESOC). http://www.oculab.com/ficheros/ojoseco.pdf. Accessed July 19, 2018.

11. Viso E, Rodríguez-Ares MT, Abelenda D, Oubiña B, Gude F. Prevalence of asymptomatic and symptomatic meibomian gland dysfunction in the general population of Spain. Invest Ophthalmol Vis Sci. 2012; 53(6):2601-2606. 